Годишњак Филозофског факултета у Новом Саду, Књига XLVI-3 (2021)

Annual Review of the Faculty of Philosophy, Novi Sad, Volume XLVI-3 (2021)

Frano Vrančić*

Département d'études françaises et

francophones

Université de Zadar
UDK: 821.133.1-1.09 Damas L.G.

DOI: $10.19090 /$ gff.2021.3.231-244

originalni naučni rad

\title{
LE THÈME DE L'IDENTITÉ DANS LA POÉSIE DAMASSIENNE
}

Cet article se donne pour tâche d'analyser le thème de l'identité dans l'œuvre poétique de l'un des pères fondateurs des lettres afro-antillaises Léon-Gontran Damas (19121978), dont les ouvrages sont toujours mieux connus dans les pays anglophones ou lusophones qu'en France métropolitaine. En partant du premier cri de révolte noire en poésie contre la présence française dans l'outre mer (Pigments, 1937), nous allons tenter d'expliquer l'originalité de cet écrivain aux multiples facettes dont les prises de positions contre les politiques assimilationnistes de la République française et les conséquences désastreuses de l'entreprise coloniale ont longuement irrigué la pensée des auteurs créoles (Tirolien, Fanon, Glissant, Depestre) dans leur combat pour une meilleure prise en compte des spécificités culturelles et linguistiques des Antilles françaises.

Mots clés : poésie, identité, Damas, Négritude, racisme, traite, assimilation, colonialisme.

\section{INTRODUCTION}

Auteur de la première anthologie des poètes noirs et jaunes (Poètes d'expression française, 1947), figure emblématique des études postcoloniales, conférencier dans les universités les plus connues des États-Unis, député de la circonscription de la Guyane au Palais Bourbon, l'un des pères fondateurs de la Négritude et de l'Antillanité, chantre de l'universalisme, délégué de la Société Africaine de Culture (SAC) à l'Unesco ou simplement Léon-Gontran Damas. Force est cependant de constater qu'en raison de l'ampleur et du retentissement de l'oeuvre césairo-senghorienne beaucoup moins d'études ont été consacrées au plus noir des trois fondateurs de la Négritude. La question qui se pose d'emblée est donc d'expliquer les raisons de cet ostracisme et de

\footnotetext{
*franevrancic@yahoo.com
} 
rendre à Damas ce qui est à Damas, car, faut-il le souligner, ses œuvres littéraires, introuvables dans les bibliothèques hexagonales il y a encore une dizaine d'années, constituent toujours la meilleure défense contre les tentatives d'archipélisation des sociétés anciennement esclavagistes et la banalisation d'une parole raciste débridée au sein de la sphère publique occidentale. Cependant, pour bien démontrer toute actualité de la poésie damassienne et mieux saisir ce contre quoi elle s'insurge il importe surtout de décrire le contexte politique, culturel et social de Paris des années 1930 et l'impact que celui-ci avait sur le sursaut de prise de conscience noire et anticoloniale du jeune étudiant nécessiteux, réfractaire à toute forme de paternalisme républicain.

\section{DAMAS, INITIATEUR DE LA NÉGRITUDE}

Léon Damas voit le jour le 28 mars 1912 à Cayenne au sein d'une famille où se mêlait une triple hérédité africaine, indienne et blanche. Suite aux décès de sa mère et de sa sœur jumelle, survenus l'année suivante, il perdra la parole jusqu'à l'âge de six ans et sera confié à sa tante Gabrielle, qui lui prodiguera une éducation sévère et bourgeoise avant de l'envoyer poursuivre ses études au lycée Schoelcher de Fort-de-France. Or, après la mutation de son père Ernest dans une société de travaux publics en 1928, c'est au collège de Meaux (Île-de-France) qu'il s'inscrit et qu'il s'érige pour la première fois en défenseur de sa race opprimée. Car à la question que lui posait le directeur de l'établissement, à savoir si le jeune Damas était le fils d'un forçat, celui-ci lui répondait que si cela était le cas, il ne serait pas Noir, mais aussi blanc qu'un Français de souche. Face au racisme décomplexé da la bourgeoisie francilienne et le déni pur et simple de la culture africaine, le jeune Guyanais se rend mieux compte des effets destructeurs de la pensée unique occidentale et de la nocivité de la politique coloniale d'assimilation. C'est pourquoi Damas se met à fréquenter les surréalistes, le panafricaniste haïtien Léo Sajous et les Martiniquaises Jeanne et Paulette Nardal, grandes devancières du mouvement de la négritude qui accueillaient des rencontres des intellectuels noirs anglophones de la génération de Harlem avec leurs homologues francophones dans leur salon parisien et dont la La Revue du Monde Noir avait pour but de donner aux étudiants colonisés l'espace où ils peuvent illustrer les richesses de la civilisation négro-africaine en toute tranquilité. Toutefois, cette revue cesse de paraître après seulement six numéros, ce qui incite Damas à chercher 
d'autres tribunes pour exprimer son refus catégorique de la doxa coloniale républicaine. Et il les trouve provisoirement dans Légitime défense, revue marxisante dont la ligne éditoriale insiste à ce que la révolution politique précède la révolution culturelle, et dans L'Étudiant noir, organe des étudiants coloniaux dont Damas était le secrétaire de rédaction et dans lequel le concept de négritude apparaît pour la première fois sous la plume de Césaire. À en croire Damas,

notre objectif était triple : tout d'abord, nous cherchions à réhabiliter la race noire et à la délivrer du terme péjoratif de 'nègre'; ensuite, nous voulions dénoncer le système colonial, en particulier le principe sur lequel il reposait, à savoir, l'assimilation par l'éducation, l'aliénation des individus et leur dépersonnalisation. Enfin, il nous fallait liquider le préjugé de couleur et de décolonisation (Racine, 1983 : 196).

Pour y parvenir, les pères de la négritude s'inspirent de l'écriture surréaliste, dont Damas se fait surtout l'apôtre, comme elle leur permet de mettre au jour le subconscient le plus refoulé et d'assumer une liberté totale contre les contraintes civilisatrices d'une République bienfaitrice qui aurait apporté les Lumières aux colonisés. Profondément influencé par les attaques surréalistes contre la cupidité et l'ethnocentrisme des classes dominantes européennes, Damas joue un rôle de premier plan dans la genèse de la négritude car il aborde, avant son ami martiniquais et son ami sénégalais, et de manière plus agressive, le thème de la réhabilitation du fond culturel africain et du refus de l'exploitation coloniale. Même si Césaire a employé le mot " négritude » en premier dans son long poème surréaliste Cahier d'un retour au pays natal (1939) et si Senghor l'a élaboré comme idéologie dans l'immèdiat aprèsguerre, force est de reconnaître qu'en imposant les thèmes classiques du procès de l'Occident, de l'esclavage, de la déportation, du racisme colonial, de la souffrance et de la révolte des Noirs, Damas a littéralement baptisé la négritude et que la publication de Pigments (1937) a joué un rôle majeur pour ce sursaut de prise de conscience identitaire des peuples anciennement coloniaux. Preuve, s'il en était encore besoin, que cet ouvrage détracteur sera interdit et saisi pour atteinte à la sûreté de l'État à la veille du second conflit mondial. Puisque, rappelons-le, son appel aux tirailleurs sénégalais « de foutre aux Boches la paix » (Damas, 2018 : 80) n'est pas resté sans suite en Côte d'Ivoire lorsque les jeunes soldats Ivoiriens refusaient d'être sacrifiés sur l'autel des intérêts économiques et politiques d'une Métropole lointaine. 


\section{LA PRISE DE CONSCIENCE IDENTITAIRE DANS LA POÉSIE DAMASSIENNE}

Tous les commentateurs s'accordent à dire que Pigments est son livre de poésie le plus connu dont la parution a attiré beaucoup d'attention auprès du public lettré grâce à la nouveauté des sujets développés. Encore que Damas n'y fait pas un exposé théorique et n'emploie pas le terme "négritude », le titre même du premier manifeste de cette poésie révolutionnaire en dit long sur l'importance de la place qu'occupe le thème de l'identité dans son esprit. Cependant, nous prévient Daniel Racine, « ce qui ressort de l'ensemble de ce recueil, c'est l'attitude révoltée d'un jeune contestataire noir qui découvre son identité après s'être demandé qui il est et ce qu'on voudrait qu'il fût » (Racine, 1983 : 58). Outre cette quête dramatique des origines, les poèmes de Pigments nous parlent des effets néfastes du colonialisme sur le psyché des peuples dominés. De ce fait, tout au long des vers les lecteurs assistent à une confrontation de deux univers antagonistes, celui du monde occidental et de son antipode antillo-guyanais, de l'Occident et de l'Afrique, des békés et des colonisés, du maître et de son esclave, du « je » et des « ils ». Cet avis est partagé par la grande pionnière des études africaines Lilyan Kesteloot qui stipule que la poésie damassienne naît du sentiment racial et du rejet de « tout ce que l'Europe lui avait fait ingurgiter de force, à lui et à ces ancêtres » (Kesteloot, 2004 : 113). Le poème liminaire du recueil (« Ils sont venus ce soir ») est très révélateur à cet égard, car l'auteur y décrit remarquablement bien toute inhumanité des esclavagistes et de leur razzias, lesquelles ont entraîné la mort et la zombification des centaines de milliers de Noirs dans le Nouveau Monde. D'origine guyanaise, Damas est parfaitement dans son rôle lorsqu'il dénonce les méfaits de la " tâche de l'homme blanc », comme il sait par expérience personnelle que le péché originel du colonialisme ne fut pas tellement l'esclavage mais la traite transatlantique, c'est-à-dire cet arrachement du continent noir des hommes, des femmes et des enfants, jetés dans les navires négriers et transportés depuis les ports occidentaux (Nantes, Bordeaux, etc.) aux Amériques pour en faire des objets. C'est ce qui lui permet de dresser son glaçant constat contre cette mission dé-civilisatrice et le scandaleux devoir des races supérieures à civiliser les races inférieures : " DEPUIS/ combien de MOI MOI MOI/ sont morts/ depuis qu'ils sont venus ce soir où le/ tam/ tam/ roulait de/ rythme/ en rythme/ la frénésie » (Damas, 2018 : 13). Il va de soi que le rôle néfaste de la civilisation occidentale dans la sujétion psychologique des Noirs et l'acculturation des sociétés indigènes ne 
sera pas sans impact sur l'instigateur de la négritude, puisque les thèmes de la question raciale et du combat contre les valeurs blanches seront toujours ses sujets de prédilection. Pour dire la vérité, Damas ne voit que le bien dans l'être noir pendant ses années estudiantines. Ce phénomène, que Sartre définit comme " racisme antiraciste » dans son Orphée noir, est sans doute une réponse aux siècles de racisme des puissances coloniales et de leur littératures respectives. Certes, Damas n'est pas conscient de son racisme de jeunesse. La meilleure preuve en est son poème "Limbé » (en langue bantoue " chagrin amoureux ») où sa glorification de la beauté de la femme noire est basée exclusivement sur la couleur de sa peau. Bien plus, il ressent un tel désir de se venger des humiliations subies par les femmes de sa race qu'il traite les femmes françaises avec le plus grand dédain. Raison pour laquelle il oppose ses «poupées noires » (Damas, 2018:43) aux prostituées parisiennes parce que seule une femme générique est capable de chasser les images obsédantes de celles-ci et de lui apporter une consolation dans son exil :

\author{
Rendez-les-moi mes poupées noires \\ qu'elles dissipent \\ l'image des catins blêmes \\ marchands d'amour qui s'en vont viennent \\ sur le boulevard de mon ennui
}

Rendez-les-moi mes poupées noires

qu'elles dissipent

l'image sempiternelle

l'image hallucinante

des fantoches empilés fessus

dont le vent porte au nez

la misère miséricorde (Damas, 2018 : 43).

En établissant une telle hiérarchie raciale Damas s'inscrit dans le mouvement de ses illustres prédécesseurs de la Harlem Renaissance qui plaçaient eux aussi le noir au sommet de la vie. En ce sens, il n'est pas inutile de rappeler que cette vision de la femme africaine a grandement inspiré Senghor pour la rédaction de sa célèbre « Femme noire » (Chants d'ombre, 1945), poème qui reste le plus bel hommage à la beauté intemporelle des femmes africaines à ce jour. C'est ce que le préfacier de Pigments Robert Desnos a très bien vu en soulignant le fait que « Damas est nègre » et qu'il « tient bien à sa qualité de nègre » (Desnos, 1937: 1). Et s'il rejette en bloc les vêtements d'emprunt imposés par la civilisation occidentale, c'est pour mieux les substituer avec ceux de la culture 
afro-guyanaise. D’où son refus catégorique de l'assimilation, quel soit de nature éthique, éducative, religieuse, vestimentaire ou alimentaire. Ainsi, dans " Hoquet ", poème le plus commenté de Pigments, Damas ridiculise cette assimilation culturelle qui consiste à imiter le modèle français jusqu'au moindre détail, de telle sorte qu'un écolier indigène doive assimiler même la nourriture à la façon occidentale ou bien choisir ses fréquentations en fonction de leur appartenance religieuse :

Ma mère voulant d'un fils très bonnes manières à table

Les mains sur la table

le pain ne se coupe pas

le pain se rompt

le pain ne se gaspille pas

le pain de Dieu

le pain de la sueur du front de votre Père

le pain du pain. (Damas, $2018: 35$ )

\section{$[\ldots]$}

Vous n'avez pas salué voisine

encore vos chaussures de sales

et que je vous y reprenne dans la rue

sur l'herbe ou la Savane

à l'ombre du Monument aux Morts

à jouer

à vous ébattre avec Untel

avec Untel qui n'a pas reçu le baptême. (Damas, 2018 : 37)

Le titre même du poème renvoie aux troubles mentaux des colonisés qui ressentent du dégoût face aux règles étouffantes de la culture dominante et aux complexes d'infériorité que les maîtres blancs essaient de leur inculquer. Ce hoquet serait donc une maladie incurable, un fardeau que chaque indigène doit porter sa vie durant. Car rien ne peut le soulager, vu la complexité et la profondeur du problème. Or le poète se révolte contre l'éducation très autoritaire de sa mère adoptive qui, en prônant le mépris contre son peuple et ses valeurs culturelles, devient idiote utile des colonisateurs et instrument puissant de son avilissement. Inspiré par la lecture du roman Banjo (1929) du romancier afro-américain Claude MacKay, l'étudiant marxisant et futur député socialiste (S.F.I.O.) refuse également la religion chrétienne et l'Église catholique de France, comme il croit qu'elles ont depuis bien trop longtemps justifié 
l'exploitation coloniale et la dépersonnalisation des descendants d'anciens esclaves qui s'en est suivie. La faute en reviendrait aux missionnaires ayant appliqués à la règle le "Code noir » de Colbert dont le véritable but selon lui était de christianiser les Noirs pour qu'ils perdent envie de se révolter et acceptent finalement leur triste sort avec résignation. Conséquemment, on ne doit pas être surpris si Damas n'a pas envie d'aller à la messe le dimanche et de faire des signes de croix après ses repas de midi. Comme l'a bien vu le romancier franco-guadeloupéen Daniel Maximin, « ainsi, dès l'origine, Damas est CONTRE. Contre la colonisation, les préjugés raciaux, l'exil inhospitalier, l'acculturation, l'assimilation, mais aussi la soumission, le mimétisme, les fauxsemblants, l'aliénation complaisante, les masques blancs de lui-même et des siens. Contre EUX » (Damas, $2014:$ 19). C'est ce qu'il démontre à merveille dans " Blanchi », "Si Souvent » et " Pour sûr », pièces où la nausée que lui inspire cette civilisation dominatrice se transforme en une véritable haine. Le remords que Damas ressent à cause de l'éducation bourgeoise reçue et ses compromissions avec le projet assimilationniste républicain le taraudent à tel point qu'il cherche à anéantir son personnalité d'emprunt faisant de lui le complice de son propre assujettissement avec des mains « effroyablement rouges du sang de leur ci-vi-li-sa-tion » (Damas, 2018: 42) :

je me sens prêt à écumer de rage

contre ce qui m'entoure

ce qui m'empêche

à jamais d'être

un homme

Et rien

ne saurait calmer autant ma haine

qu'une belle mare de sang

faite

de ses coutelas tranchants

qui mettent à nu

les mornes à rhum. (Damas, 2018 : 49)

Ou encore :

Alors

je vous mettrai les pieds dans le plat

ou bien tout simplement

la main au collet

de tout ce qui m'emmerde en gros caractères

colonisation 
civilisation

assimilation

et la suite. (Damas, $2018: 53$ )

Ses recueils d'après-guerre sont du même acabit, excepté Graffiti (1952), son seul livre de poésie d'inspiration amoureuse dans lequel le poète s'adresse à une femme non-identifiée de sa race qui est censée apporter la paix et la sérénité à son âme tourmentée. Cependant, il y a une pièce de Graffiti (" Comme un rosaire »), reprise dans Névralgies (1966), où sa satire des autorités ecclésiastiques et sa vision lutte des classes retient toute notre attention. À l'opposé de Senghor qui arrive à pardonner à la France coloniale dans sa très catholique Prière de paix (Hosties noires, 1948), Damas éprouvera toujours de la rancune à l'égard de l'Église de France à tel point qu'il la considérera toujours comme suppôt le plus fidèle de la conquête coloniale et la grande responsable de l'aliénation psychique des indigènes. C'est ce qui explique en partie l'irrévérence de ses prises de position contre les prêtres, qu'il culpabilise toujours dans sa poésie et ce même pour avoir prétendument « coupé leur sexe aux nègres pour en faire des bougies pour leurs églises » (Damas, 2018 : 52). Bien évidemment, « Comme un rosaire » n'est pas sans rappeler sa pièce « Il me souvient encore » de Graffiti où l'écrivain se remémore de l'expérience décevante qu'était sa première communion et où il traite son curé « du sorcier en soutane » (Damas, $2018: 86)$ :

\author{
COMME UN ROSAIRE \\ s'égrène \\ pour le repos \\ d'une âme \\ mes nuits \\ s'en vont par cinq \\ dans un silence \\ de monastère \\ hanté. (Damas, 2018 : 93)
}

Dans la même veine, il convient encore de préciser ici qu'en dépit de l'omniprésence du thème de l'amour déçu on retrouve dans Névralgies la haine toujours grandissante des institutions bourgeoises et des élites de couleur propre à l'auteur de Pigments :

Vous dont la présence proche ou lointaine 
énerve ma vie

comme la vieille folle du coin

mon premier sommeil

Vous dont le crime est d'en vouloir

à l'image

qu'il m’a plu

d'avoir un matin

d'Elle

Vous dont les ricanements

vous dont le visage

vous dont le cœur

vous dont la présence

vous dont le crime

Et puis vous tous

enfin vous autres

saisirez-vous jamais un rien même

à ce poème

mon drame. (Damas, 2018 : 106-107)

Le thème de l'identité prédomine toujours dans son chef-d'oeuvre poétique Black-Label (1956), petit poème en quatre parties publié chez Gallimard et dont le titre renvoie non seulement à une marque de whisky que l'écrivain boit pour soulager sa peine mais aussi à la couleur de sa race opprimée. Damas y évoque son mal du pays, responsable en partie pour son isolement social, et parle de sa Guyane natale avec beaucoup de nostalgie et de fierté. Ainsi, dans son esprit cette « Terre des Parias » n'est pas un bagne, mais un royaume d'enfance où le poète-député cayennais retrouve ses repères perdus pour mieux combattre le spleen et la domination idéologico-culturelle de l'Occident. Dès lors, il revendique haut et fort sa guyanité en mettant en valeur surtout le fait que le brassage des races et des cultures différentes (Noirs, Blancs, Amérindiens) coule dans ses veines, comme trois fleuves. Encore une fois, comme à son habitude, Damas n'est pas tendre avec ses compatriotes, qui partagent eux aussi une grande part de responsabilité pour leurs propres malheurs. Car en acceptant la théorie de la « table rase » et de la « tâche civilisatrice » ils trahissent la culture de leurs ancêtres et se contentent de vivre dans le mensonge et l'humiliation permanente. C'en est de même des victimes de la traite que l'auteur prend à partie pour leur manque de combativité et une si facile acceptation de traverser l'océan dans 
des conditions inhumaines après avoir été marquées au fer rouge et parquées dans des embarcations sordides. Il en veut également aux femmes qui se sont non seulement prostituées avec leurs bourreaux mais ont encore dénoncé ceux des leurs qui ont tenté de se révolter (Racine, 1983 : 118).

Antonella Emina a donc parfaitement raison de dire que la poésie damassienne désigne d'une part « l'assimilation comme le danger le plus exécrable, ridicule à la limite du grotesque, et, d'autre part, en souligne l'ignominie parce qu'obtenue avec la connivence de l'homme dominé » (Emina, 2018 : 150). Né du déracinement culturel et de l'assimilation outrancière à la culture française, son amertume contre l'éducation bourgeoise des élites guyanaises éclate ici de nouveau à grand jour car, à la différence de ses cousins de Rémire qui « parlaient si librement patois/ sans crainte d'être jamais mis au pain sec/ ni jetés au cachot » (Damas, 2011: 63), le poète continue à vociférer avec la même virulence contre le maître et l'école pour mieux exalter « les rebelles/ les réfractaires/ les cul-terreux/ les insoumis/ les vagabonds/ les bons absents/ les propres à rien » (Damas, 2011 : 67), bref, tous ceux et celles qui refusent toute idée de chosification de la personnalité noire. Signalons néanmoins que sa foi en le renouveau de la race noire reste intacte malgré le pessimisme ambiant de Black-Label. Et s'il croit pouvoir soutenir que la victoire de la race des parias est à portée de main, c'est que les Blancs commencent à se passionner pour l'art nègre, comme en témoigne l'engouement des milieux artistiques parisiens de l'époque pour la danse et la musique des pays africains :

PARIS-Nombril-du-Monde

à la merci de l'Afrique

de sa voix

de ses regrets

de sa joie

ses tristesses

à la merci de la fièvre du rythme

de la piste un mouchoir de poche

de l'invitation au voyage aux murs

de la trompette bouchée

on eût dit Celle du JUGEMENT DERNIER

Le Blanc à l'École du Nègre. (Damas, 2011 : 52-53) 
C'est pourquoi Damas rend un vibrant hommage au continent noir et à la grande civilisation africaine avant de conclure que jamais les Blancs ne seront en mesure d'assimiler les attributs inhérents aux peuples de couleur, attributs qui doivent devenir une source de fierté pour les Noires s'ils veulent recouvrer leur identité bafouée :

\author{
ET BLACK-LABEL À BOIRE \\ pour ne pas changer \\ Black-Label à boire \\ à quoi bon changer. (Damas, $2011: 68$ )
}

Aussi importe-t-il de noter qu'on retrouve cette même fierté identitaire dans son recueil Dernière escale (2012), publié à titre posthume à l'occasion du centenaire de sa naissance et dont la lecture nous permet de mieux comprendre l'évolution du poète cayennais pendant ses années américaines. Outre les thèmes de l'amour de la femme noire, ces poèmes démontrent une nouvelle fois son inébranlable attachement à la cause noire et aux terres guyanaises, comme l'illustre bien le souvenir du « placenta planté/ toujours enfoui dans l'arrière-cour de la Maison où fut le bananier sacré » (Damas, 2012 : 44) et les savanes de Matiti qui sont à leur tour des endroits privilégiés de la transmission des valeurs noires aux enfants du pays. Soucieux de secouer tous les vasselages, son mépris des valeurs capitalistes et du christianisme réapparaît avec force, notamment dans les poèmes « Puisque » et « Passée la vacherie des temps » où Damas réitère ses accusations contre les autorités républicaines d'avoir utilisé les autochtones comme chair à canon lors des deux conflits mondiaux et contre l'Église de France dont la " croix/ chrétienne/ catholique et romaine et latine et françoise/ de guerre/ de fer/ de bois» (Damas, 2012: 109) serait tachée du sang d'innocents. Seuls les marrons, martèle-t-il, ont réussi à se sauver des « bienfaits de la tâche civilisatrice » en prenant la fuite dans les forêts vierges amazoniennes. Comme dans son essai subversif Retour de Guyane (1938), Damas rend ici un hommage appuyé à ces populations de l'intérieur guyanais, qui n'ont pas rompu le cordon ombilical avec la Mère-Afrique et qui sont les seuls à pouvoir transformer en profondeur le pays. Étant foncièrement insoumis au diktat de la civilisation occidentale, il se permet de les proposer en exemple à tous les indigènes parce qu'il est fermement convaincu que leurs coutumes, contes, carnavals et bals devraient guérir les Noirs de leur dépersonnalisation culturelle, conditio sine qua non 
d'une éventuelle libération politique. C'est ce qui explique son besoin de relancer «le thème de la rébellion individuelle » dans les pièces conclusives du recueil dans lesquelles le substantif " fin », contrairement au poème final de Névralgies, «n'a donc rien à voir avec tout ce qui concerne la fin, voire le figé, le dépassé, l'agonie, la mort » mais annonce plutôt « la première action du nouvel ordre appelé par De la profuse et diffuse odeur fauve » (Emina, 2018 : 98).

\section{CONCLUSION}

En guise de conclusion nous dirons que le rôle damassien dans la prise de conscience identitaire des peuples anciennement colonisés et la genèse des littératures noires de langue française n'est plus à démontrer. Car, comme l'a rappelé très justement son compagnon de route guadaloupéen et l'un des cofondateurs du mouvement négritudien Guy Tirolien, "Damas était la négritude en action. Il fut le premier d'entre nous à pratiquer les Noirs américains, les écrivains surtout. [...] Si bien que, par Damas nous avions une perspective sur la société noire américaine et sur l'inteligentzia noire » (AlanteLima, 1991 : 185). Son parcours,

déterminé par le déracinement, commence avec la reconnaissance de deux civilisations - la civilisation occidentale, voire française, et celle hybride développée en pays colonisé - dont l'antagonisme est ferme dès le début et ne s'estompe pas avec le temps. Cette connaissance de l'un et de l'autre se fait surtout à travers les regards réciproques sur les petites choses du quotidien, telles que la nourriture, les habitudes vestimentaires, la langue utilisée, etc (Emina, $2018:$ 149).

Tiraillé entre deux cultures et victime quotidien de discriminations racistes, Damas dénoncera toujours avec la même véhémence l'exploitation coloniale et les politiques assimilationnistes sur lesquelles la " République coloniale » s'appuie pour dominer les populations ultramarines de son empire. Nullement surprenant donc si le poète franco-guyanais s'acharnera tout au long de sa vie à mieux revaloriser les acquis de la civilisation noire et à éradiquer les complexes d'infériorité que l'esclavage et la " mission civilisatrice » ont durablement imprimé à la conscience des indigènes. Animé d'une passion indéfectible pour « la Nation de Paria » (Damas, 2012 : 25), il n'aura de cesse de fustiger le préjugé de couleur et l'esprit mulâtre des élites guyanaises en leur faisant comprendre que seul le refus pur et simple de la culture européenne pourrait les aider à se libérer de la haine de soi savamment inculquée par les 
tenants de la doxa coloniale. C'est dire qu'à l'heure des crispations identitaires la poésie damassienne demeure toujours d'actualité, car elle permet aux Français ultramarins d'être eux-mêmes sans se fermer à l'Autre et d'inviter les « vieux Français » de sortir de leurs prisons identitaires afin de pouvoir participer au "rendez-vous du donner et du recevoir », pour employer la très belle formule césairienne. Ajoutons, pour terminer, qu'en dépit des mots durs que Damas a contre la mission civilisatrice et certaines affirmations exagérées adressées contre les missionnaires, comme celle selon laquelle le christianisme serait le principal responsable de l'asservissement psychologique des Noirs, tout porte à croire qu'il cherche à éviter une pensée simplifiée qui diaboliserait l'homme blanc judéo-chrétien et caresserait les Antillo-Guyanais dans le sens du poil. Comme il croit que la cause du mal soit la ségrégation raciale et la domination capitaliste, on peut dire que ses recueils de poèmes ne sont aucunement des discours haineux contre l'Occident, mais des appels à l'amour et à la solidarité de tous les opprimés du monde, notamment des salariés coloniaux, ce dont il est surtout question dans la préface desnosienne de Pigments :

Ils sont à la gloire, ces poèmes, de tout l'immense prolétariat indigène des colonies. Ils nous signifient que le temps est venu de poursuivre la conquête de ces terres et de ses peuples. Ne sont-elles pas exploitées comme les nôtres, ces terres. Et ces peuples ne sont-ils pas... voyez un peu où la plume et le bon sens nous entraînent ! Ces poèmes sont donc aussi un chant d'amitié offert, au nom de toute sa race, par mon ami, le nègre Damas, à tous ses frères blancs. Un don de la savane à l'usine, de la plantation à la ferme, de la fabrique tropicale à l'atelier européen. (Desnos, $1937:$ 1)

Frano Vrančić

THE THEME OF IDENTITY IN LÉON-GONTRAN DAMAS'S POETRY

\section{Summary}

This article analyzes the theme of identity in the poetic work of one of the founding fathers of Afro-Caribbean letters Léon-Gontran Damas (1912-1978), whose works are still better known in English-speaking or Portuguese-speaking countries than in metropolitan France. Starting from the first cry of black revolt in poetry against the French presence in the overseas territories (Pigments, 1937), we will try to explain the originality of this multifaceted writer whose positions taken against the assimilationist policies of the French Republic and the disastrous consequences of the colonial 
enterprise have long irrigated the thought of Creole authors (Tirolien, Fanon, Glissant, Depestre) in their fight for a better consideration of the cultural and linguistic specificities of the French Antilles. In addition, the paper pays homage to his extraordinary literary work as well as his struggle for the freedom and the dignity of the colored people worldwide. What is more, the rise in power of racist and discriminatory attacks and the extremization of the discourse of many Western politicians prove that the author's literary work has never been so topical since it still constitutes a bulwark against the resurrection of European colonial thought and its imperialist excesses. After all, Damas' anti-racism invites us to fiercely resist a logic of scapegoating minorities in multicultural societies, especially in formerly colonizing and slaving countries where the followers of the exploitation of man by man occupy the center front of the media scene and whose hate speech against socially disadvantaged classes seriously undermine living together.

Key words: poetry, identity, Damas, negritude, racism, trafficking, assimilation, colonialism.

\section{RÉFÉRENCES BIBLIOGRAPHIQUES}

Alante-Lima, W. (1991). Guy Tirolien l'homme et l'oeuvre. Paris : Présence Africaine.

Damas, L.-G. (2011). Black-Label et autres poèmes. Paris : Gallimard.

Damas, L.-G. (2012). Dernière escale. Paris : Le Regard du texte, Atelier Vincent Auger.

Damas, L.-G. (2018). Pigments Névralgies. Paris : Présence Africaine.

Emina, A. (2018). Léon-Gontran Damas Les détours vers la Cité Neuve. Paris: L'Harmattan.

Emina, A. (sous la dir.) (2014). Cent ans en noir et blanc. Paris : CNRS Éditions.

Kesteloot, L. (2004). Histoire de la littérature négro-africaine. Paris : KarthalaAUF.

Maximin, D. (2014). Léon Damas, étoile pigmentée de Graffiti. Cent ans en noir et blanc. Paris : CNRS Éditions, 15-25.

Racine, D. (1983). Léon-Gontran Damas l'homme et l'oeuvre, préface de L. S. Senghor. Paris : Présence Africaine. 